\title{
ISOMETRIES OF A FUNCTION SPACE
}

\author{
U.D. VYAS \\ Department of Mathematics and Computer Science \\ Winston-Salem State University \\ Winston-Salem, North Carolina 27110 \\ (Received July 24, 1984 and in revised form February 16, 1985)
}

ABSTRACT. It is proved here that an isometry on the subset of all positive functions of $L^{1} \cap L^{p}(\mathbb{R})$ can be characterized by means of a function $h$ together with a Borel measurable mapping $\phi$ of $\mathbb{R}$, thus generalizing the Banach-Lamparti theorem of $L^{\mathrm{p}}$ spaces.

KEY WORDS AND PHRASES. Borel measure, function space, Banach-Lamparti Theorem. 1980 AMS SUBJECT CLASSIFICATION CODE. 46E

1. INTRODUCTION.

Edwards [1] proves that all bipositive isomorphisms of $\mathrm{L}^{\mathrm{p}}(1 \leqq \mathrm{p}<\infty)$ convolution algebras of a compact group are induced by bicontinuous isomorphisms of the group. By changing the algebra isomorphism from bipositive to an isometry Strichartz [2] establishes the same type of result with the exception of $p=2$. Here we consider isometries of $L^{1} \cap L^{p}(\mathbb{R}), p \neq 2$, and give a general form to the Banach-Lamparti theorem proving the isometry equivalent to a combination of a function $h$ and a Borel measurable mapping $\phi$ of $\mathbb{R}$.

2. THE ELEMENTARY LEMMAS.

The norm of a function in $L^{1} \cap L^{p}(\mathbb{R})$, denoted by $\|f\|_{n}$, is defined by

$$
\|f\|_{n}=\|f\|_{p}+\|f\|_{1}
$$

A condition equivalent to the equality of norms of $f+g$ and $f-g$ for positive functions of $L^{1} \cap L^{p}(\mathbb{R})$ is given in the following lemma.

LEMMA 1. Let $f, g \in L^{1} \cap L^{p}(\mathbb{R})$ and $f, g \geqq 0$. Then

$$
\|f+g\|_{n}=\|f-g\|_{n} \Leftrightarrow f \cdot g \cdot=0 \text { a.e. }
$$

PROOF. From Royden [3] we have

$$
\|f+g\|_{p}^{p}+\|f-g\|_{p}^{p}=2\left(\|f\|_{p}^{p}+\|g\|_{p}^{p}\right) \Leftrightarrow f g=0 \text { a.e. }
$$

Now, $\|f+g\|_{p}^{p}=\int(f+g)^{p}=\int f^{p}+\int g^{p} \quad \Leftrightarrow f g=0$.

Thus, $\|f+g\|_{p}^{p}=\|f\|_{p}^{p}+\|g\|_{p}^{p} \quad \Leftrightarrow \quad f g=0$. 
From (2.1) and (2.2), we get

or

$$
\begin{aligned}
& \|f+g\|_{p}^{p}=\|f-g\|_{p}^{p} \Leftrightarrow f g=0 \\
& \|f+g\|_{p}=\|f-g\|_{p} \Leftrightarrow f g=0
\end{aligned}
$$

In particular for $p=1 \quad(2.3)$ becomes

$$
\|f+g\|_{t}=\|f-g\|_{1} \Leftrightarrow f g=0
$$

Addition of (2.3) and (2.4) yields

$$
f g=0 \quad \rightarrow \quad\|f+g\|_{n}=\|f-g\|_{n} \text {. }
$$

Conversely, if $\|f+g\|_{n}=\|f-g\|_{n}$, then

$$
\left(\|f+g\|_{p}-\|f-g\|_{p}\right)+\left(\|f+g\|_{1}-\|f-g\|_{1}\right)=0 \text {. }
$$

Since both of the terms in parentheses are positive, we obtain $f g=0$ by (2.3) and $(2.4)$.

In the next lemma we show that for positive functions if $L^{1} \cap L^{p}$ (R) on isometry preserves the disjointness of supports.

LEMMA 2. Let $f, g \in L^{1} \cap L^{p}$ and $f, g \neq 0$. Let $u$ be an isometry on $L^{1} \cap L^{p}$. Then

$$
\text { (Supp f) } \cap(\text { Supp g) }=\phi \Leftrightarrow(\text { Supp Uf) } \cap(\text { Supp } \cup g)=\phi
$$

PROOF. (Supp f) $\cap($ Supp $g)=\phi \Leftrightarrow f g=0$

$$
\begin{aligned}
& \Leftrightarrow\|f+g\|_{n}=\|f-g\|_{n} \quad \text { (Lemma 1) } \\
& \Leftrightarrow\|u f+u g\|_{n}=\|u f-v g\|_{n} \\
& \Leftrightarrow(u f)(u g)=0 \\
& \Leftrightarrow \quad(\text { Supp vf) } n(\text { Supp } u g)=\phi .
\end{aligned}
$$

3. THE THEOREM.

Let $1 \leq \mathrm{p}<\infty, \mathrm{p} \neq 2$ and $\mathrm{U}$ be a ono-one onto linear transformation on positive functions of $L^{1} \cap L^{p}(\mathbb{R})$ such that $\|u f\|_{n}=\|f\|_{n}$. Then there is a one-one Borel measurable mapping of $\mathbf{R}$ onto itself and a function $h$ such that

$$
\text { uf }=h(f(\phi)) \text { for all positive } f \in L^{1} \cap L^{p} \text {. }
$$

PROOF. Let $M_{0}$ denote the family of sets of measure zero. Clearly $M_{0}$ is a $\sigma$-ideal of $B$, where $B$ is the family of Borel sets.

For the $\sigma$-algebra

$$
B / M_{0}=\left\{A \mid A=\text { Suppf, } f \text { positive, } f \in L^{1} \cap L^{p}\right\}
$$


Define a map

$$
\begin{aligned}
& \Phi: B / M_{0} \rightarrow B / M_{0} \text { by } \\
& \Phi(A)=\text { Supp Ue }-x^{2} X_{A} \text {, where } A=\text { Suppf. }
\end{aligned}
$$

We shall prove that $\Phi$ is an $\sigma$-isomorphism.

For this we must prove the following:

$$
\begin{aligned}
& \text { (i) } \Phi(A \cup B)=\Phi(A) \cup \Phi(B) \\
& \text { (ii) } \Phi\left(\underset{i=1}{\infty} A_{i}\right)={\underset{i=1}{U} \Phi\left(A_{i}\right)}_{\text {(iii) } \Phi(\mathbb{R})=\mathbb{R}}^{\infty} \\
& \text { (iv) } \Phi(\bar{A})=(\overline{\Phi(A)}) \quad(\bar{A}=\text { the complement of } A) \\
& \text { (v) } \Phi \text { is a bijection }
\end{aligned}
$$

(i) Let $A, B \in B / M_{O}$ and $A \cap_{B}=\phi$. Then there are $f, g \in L^{1} \cap L^{p}$ such that $A=\operatorname{suppf}$ and $B=$ suppg. So (suppf) $\cap$ (Supp $g$ ) $=\phi$. Therefore by lemma 2 we get

$$
(\text { Supp } \cup \mathrm{f}) \cap(\text { Supp } \cup g)=\phi
$$

Since $A \cap B=\phi$, we have $X_{A \cup B}=X_{A}+X_{B}$, and therefore

$$
U e^{-x^{2}} X_{A \cup B}=U e^{-x^{2}} X_{A}+U e^{-x^{2}} X_{B} \text {, (by (3.1)) }
$$

This gives,

$$
\text { Supp Ue } e^{-x^{2}} x_{A \cup B}=\operatorname{Supp} U e^{-x^{2}} x_{A}+\operatorname{supp} U e^{-x^{2}} x_{B}
$$

Thus,

$$
\Phi(\mathrm{A} \cup \mathrm{B})=\Phi(\mathrm{A}) \cup \Phi(\mathrm{B})
$$

(ii) Let $\left(A_{1}\right)_{i \in \mathbf{N}}$ be a disjoint family of members of $B / M_{0}$ and let $A=U_{i=1}^{\infty} A_{1}$, then $X_{A}=1$ im $\sum_{i=1}^{n} x_{A_{i}}$. So by linearity of $U$ we obtain

$$
\mathrm{Ue}^{-\mathrm{x}^{2} x_{A}}=\lim \sum_{i=1}^{\mathrm{n}} \mathrm{U} \mathrm{X}_{\mathrm{A}_{i}}
$$

Therefore we get

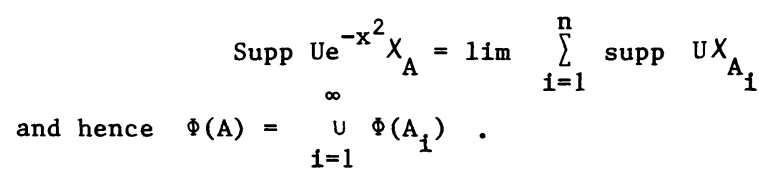

(iii) First we show that $\Phi(A) \subset \Phi(B)$ whenever $A \subset B$. For, if $A \subset B$ then, $B$ is the disjoint union of $A$ and $B-A$ and $X_{B}=X_{A}+X_{B-A}$. An application of lemma (2) gives

$$
\text { Supp Ue } e^{-x^{2}} X_{B}=\left(\text { Supp } U e^{-x^{2}} X_{A}\right) \cup\left(\text { Supp Ue } e^{-x^{2} X_{B-A}}\right)
$$


proving $\Phi(B)=\Phi(A) \cup \Phi(B-A)$ which in turn gives

$$
\Phi(\mathrm{A}) \subset \Phi(\mathrm{B})
$$

Now in order to prove $\Phi(\mathbb{R})=\mathbb{R}$, suppose that $\mathbb{R}-\Phi(\mathbb{R})=E \neq \phi$, and consider $e^{-x^{2}} X_{E} \in L^{1} \cap L^{p}$. Since $U$ is onto there exists $6 \in L^{1} \cap{ }_{L}^{p}$ such that $u \quad 6=e^{-x^{2}} x_{E}$ Therefore supp of $=$ supp $e^{-x^{2}} X_{E}=E$, giving $\Phi(A)=E$, where $A=\operatorname{supp} 6$. Thus we obtained $\mathbb{R}-\Phi(\mathbb{R})=\Phi(\mathrm{A})$ which implies $\Phi(\mathrm{A}) \notin \Phi(\mathbb{R})$, contradicting (3.2). Hence $\Phi(\mathbb{R})=\mathbb{R}$.

(iv) Since the sum of the characteristic functions on $A$ and its complement is unity we easily obtain (supp $U e^{-x^{2}} X_{A}$ ) $\cup$ (Supp $U e^{-x^{2}} X_{\bar{A}}$ ) $=$ supp $U e^{-x^{2}}$. This implies $\Phi(\mathrm{A}) \cup \Phi(\overline{\mathrm{A}})=\Phi(\mathbb{R})$ and so using (iii) we get $\Phi(\mathrm{A}) \cup \Phi(\overline{\mathrm{A}})=\mathbb{R}$. Further from $\Phi(\mathrm{A}) \cap \Phi(\overline{\mathrm{A}})=\phi$ we obtain $\overline{\Phi(\mathrm{A})}=\Phi(\overline{\mathrm{A}})$ as required.

(v) If we take $\Phi(A)=\Phi(B)$ then supp $U e^{-x^{2}} X_{A}=\operatorname{supp} U e^{-x^{2}} X_{B}$ and this implies supp $e^{-x^{2}} X_{\bar{A}} \cap$ supp $e^{-x^{2} X_{B}}=\phi$ by lemma (2). Thus $\bar{A} \cap B=\phi$ which implies $B \subset_{A}$. Interchanging the roles of $A$ and $B$ gives $A \subset B$ so that $A=B$ and thus $\Phi$ is one-one.

Now since $u$ is onto, corresponding to $e^{-x^{2} X_{A}}$, there exists $g \in L^{1} \cap L^{p}$ such that $U e^{-x^{2}} X_{A}=g$. Therefore supp $U e^{-x^{2}} X_{A}=\operatorname{suppg}$ and $\Phi(A)=\operatorname{suppg} \epsilon B / M_{0}$ proving $\Phi$ is onto.

Now it follows from a theorem of Royden [3] that $\Phi$ is a $\sigma$-isomorphism of $B / M_{0}$ onto itself. Thus there is a one-one mapping $\phi$ of $B / M_{0}$ onto itself such that $\phi$ and $\phi^{-1}$ are Borel measurable and

$$
\Phi(A)=\phi^{-1}(A) \text { modulo } M_{0} .
$$

Now, consider $X_{[0,1]} \in L^{1} \cap L^{P}$ and take $h_{1}=U\left(X_{[0,1]}\right)$. If $A_{1}$ is any Borel set of $\mathbb{R}$ contained in $[0,1]$ then $X_{[0,1]}=X_{A_{1}}+X_{[0,1]-A_{1}}$. So $h_{1}=U X_{A_{1}}+U X_{[0,1]-A_{1}}$.

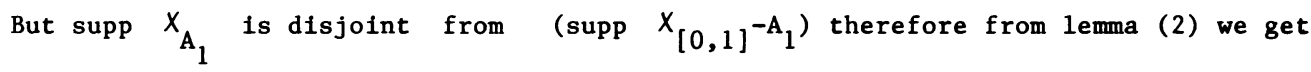

$$
\left(\operatorname{supp} \cup X_{A_{1}}\right) \cap\left(\operatorname{supp} \cup X_{[0,1]-A_{1}}\right)=\phi
$$

That is $U X_{A_{1}}$ equals $h_{1}$ on the support $u X_{A_{1}}$.

Therefore $\quad{ }^{\prime} X_{A_{1}}=h_{1} X$ supp $v X_{A_{1}}$

$$
\begin{aligned}
& =h_{1} X \text { supp } u e^{-x_{X}^{2}} A_{1} \\
& =h_{1} X \Phi\left(A_{1}\right) \\
& =h_{1}\left(X_{A_{1}} \phi\right)
\end{aligned}
$$

In general if $A_{n}$ is a Borel set contained in $[n, n+1]$ where $n \epsilon Z$, then we can have $U X_{A_{n}}=h_{n}\left(X_{A_{n}} \phi\right.$. Further if $A$ is any Borel set of $\mathbb{R}$ then there exists a Borel set of $A_{n}$ of $\mathbb{R}$ for all $n$ such that $A=\underset{n=-\infty}{U} A_{n}, A_{m} \cap A_{n}=\phi$ whenever $\mathrm{m} \neq \mathrm{n}$. 


$$
\text { Now } \begin{aligned}
u X_{A} & =u\left(X_{u_{n}}\right) \\
& =\lim _{n \rightarrow \infty} h_{n}\left(X_{A} \phi\right) \\
& =h\left(X_{A} \phi\right), \text { where } h=\lim _{n \rightarrow \infty} h_{n} \text {. }
\end{aligned}
$$

If $\psi$ is any simple function we have

$$
\mathbf{u} \psi=h(\psi(\phi))
$$

Further, functions in $L^{1} \cap L^{p}(\mathbb{R})$ can be approximated in norm by a simple function, and $U$ is norm preserving, we get

$$
u \sigma=h(f(\phi)) \text {. }
$$

ACKNOWLEDGEMENT. I express my sincere thanks to Prof. M. Rajagopalan of University of Toledo for his guidance in this work. Thanks are also due to mini grant authority of my university for partial financial support to this work.

\section{REFERENCES}

1. EDWARDS, R.E. Bipositive and Isometric Isomorphism of Some Convolution Albegra, Canad. J. Math. 17(1965), 839-846.

2. STRICHARTZ, R.S. Isomorphism of Group Algebra, Proc. Amer. Math. Soc. (1966).

3. ROYDEN, H.L. Real Analysis, The Macmillan Co., (1968). 


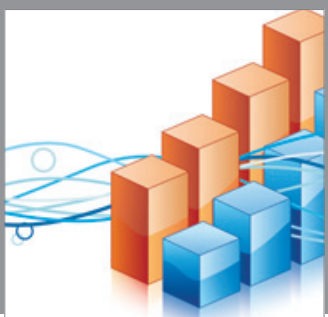

Advances in

Operations Research

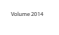

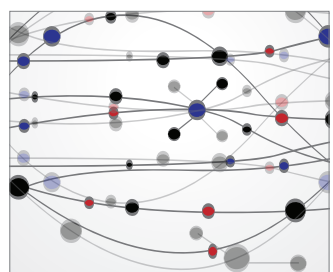

\section{The Scientific} World Journal
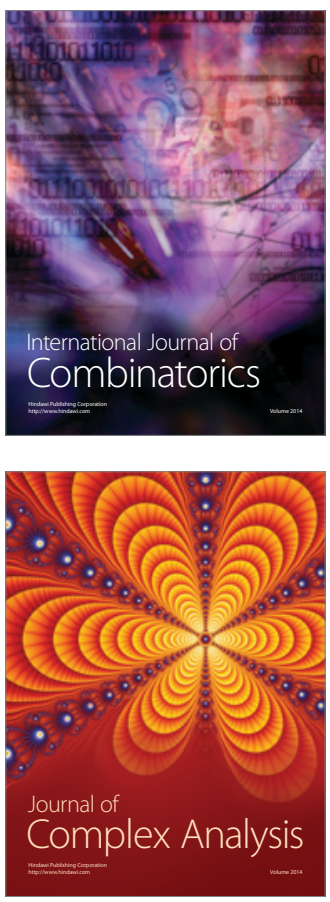

International Journal of

Mathematics and

Mathematical

Sciences
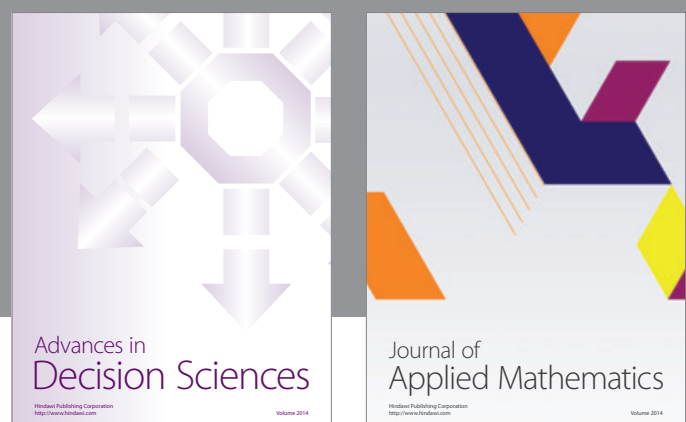

Journal of

Applied Mathematics
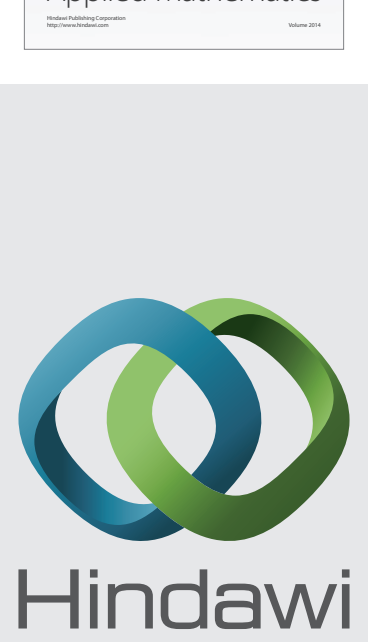

Submit your manuscripts at http://www.hindawi.com
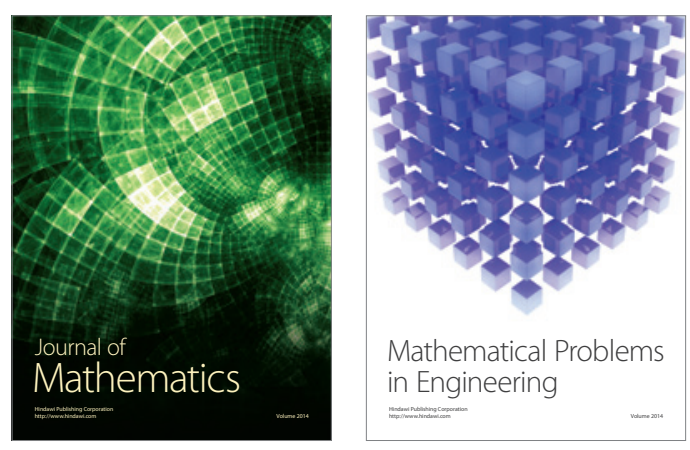

Mathematical Problems in Engineering
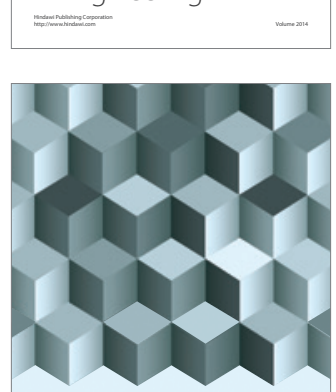

Journal of

Function Spaces
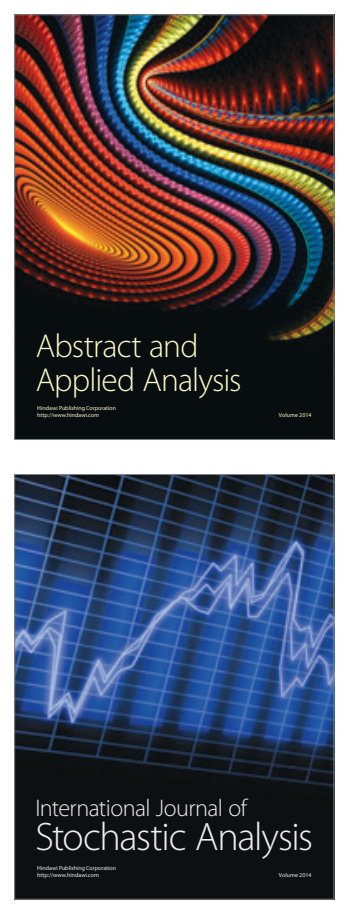

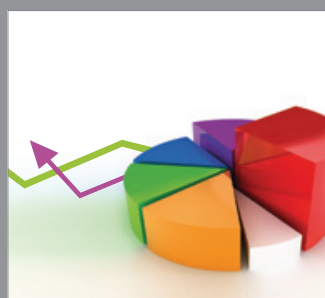

ournal of

Probability and Statistics

Promensencen
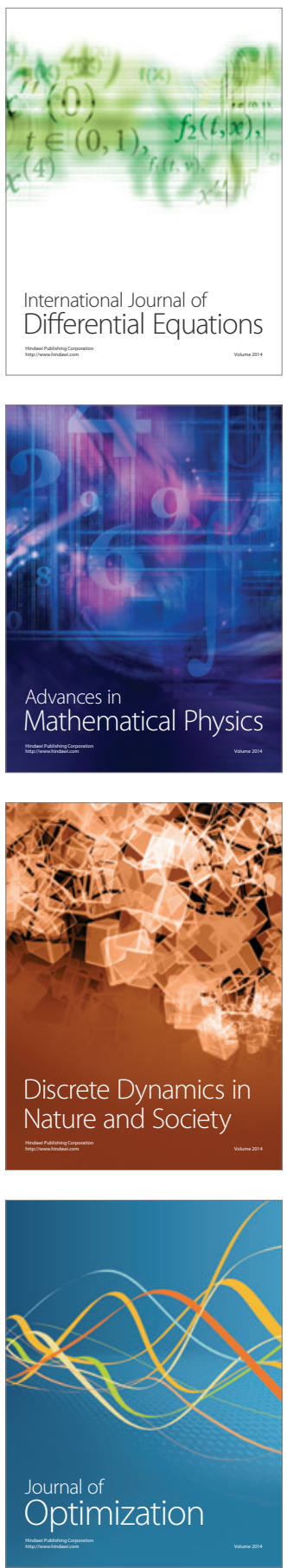